\title{
Looking at MOOCs Rapid Growth Through the Lens of Video-Based Learning Research
}

\author{
http://dx.doi.org/10.3991/ijet.v9i1.3349 \\ Michail N. Giannakos, Letizia Jaccheri and John Krogstie \\ ${ }^{1}$ Norwegian University of Science and Technology (NTNU), Trondheim, Norway
}

\begin{abstract}
Video learning is becoming an increasingly important part of contemporary education. In the decade there has been an increase of many and diverse forms of research efforts on video learning. This paper focuses on the video learning research of the last years based on 166 peerreviewed published academic papers. A categorization is then derived from these papers, delineating some basic characteristics of video learning. The taxonomy attempts to look at MOOCs rapid growth through the lens of videobased learning research. We also provide some directions for future research related to the use of video learning.
\end{abstract}

Index Terms - MOOCs, Video-based learning, Literature Review

\section{INTRODUCTION AND OBJECTIVES}

With the widespread adoption of video learning systems such as Khan Academy and edX, new research has emerged. Recent for-profit companies, such as Coursera and Udacity, have started offering forms of instruction that are primarily video-based. To date, Massive Online Open Courses (MOOCs) are offered by universities across the globe by using video lectures in many different languages and topics.

Although, the use of learning videos has been widely employed in the past years [1], to date an increased interest is observed. Millions of learners watch videos from different platforms (e.g., Opencast Matterhorn) on a diverse number of terminals (desktop, phone, tablet) [2]. Videos are also used for supervision and assessment (e.g., via Skype) and even take exams from their homes. For instance Proctor $U^{1}$, offers a service whereby a student with a webcam and microphone accesses the ProctorU website, provides proof of identification to a live proctor, and takes the test while being filmed with the webcam. In addition, MOOCs growth brought learning videos on the top of the agenda for many institutions and organizations $[3,4]$.

In the last decade, various video learning tools have been developed [5] and several empirical studies have been conducted [6] on both K-12 and higher education. To categorize the research on video learning, we reviewed past research and identified five categorization pillars. These pillars were based on the on the video technology functions and the style of use. One hundred-sixty six, peer-reviewed articles were selected from an extensive search of the literature. These articles include all the types of videos (e.g., broadcasting, on demand, slides with nar-

${ }^{1}$ ProctorU is used from many higher education institutions, like Washington State U., U. Illinois UC and Northwestern U. More information about ProctorU you can find at http://www. proctoru. com ration) used for all learning, training and even informing purposes (e.g., studying, assessing, mentoring, advertising). In particular, we analyzed the research that has been conducted in the last twelve years on video learning and in particular we investigate the following two objectives:

1. Categorizing the video learning research of the last years

2. Investigating MOOCs through the lens of videobased learning growth

This paper is structured as follows: Section II gives the proposed categorization of recent year's video learning research. Section III attempts to investigate MOOCs growth using research on video learning. Section IV provides conclusions of the paper.

\section{VIDEO LEARNING RESEARCH CATEGORIZATION}

In order to examine the research that has been conducted on video learning, an extended search on the literature was conducted. A comprehensive search of key educational and technology journals (similar to prior studies e.g., $[7,8]$ ), was completed based on a wide range of key terms including educational videos, learning videos, video lectures, webinars, (video) podcasts, video streaming, webcasts, web-videos and videocasts. Specifically, two experienced researchers searched on the following ISI indexed educational technology journals ${ }^{2}$ : Australasian Journal of Educational Technology, British Journal of Educational Technology, Computers and Education, Educational Technology Research and Development, Educational Technology and Society, Innovations in Education and Teaching International, Learning, Media and Technology and The Internet and Higher Education. After the search process, the two researchers presented their results and proposed characteristics for categorizing these studies. They discussed both the papers and the categorization pillars and they conclude into the following five:

- Portability: How portable is the video learning system?

- Synchronization: Is there any learner-tutor communication?

- Interactivity: Is the system interactive?

- Type of use: what is the type of video learning use, e.g., main or supplementary learning material?

- Context of use: In what context the video learning is performed e.g., formal, informal?

\footnotetext{
${ }^{2}$ The selection of these journals is based on prior similar reviews in the educational technology field $[7,8]$
} 


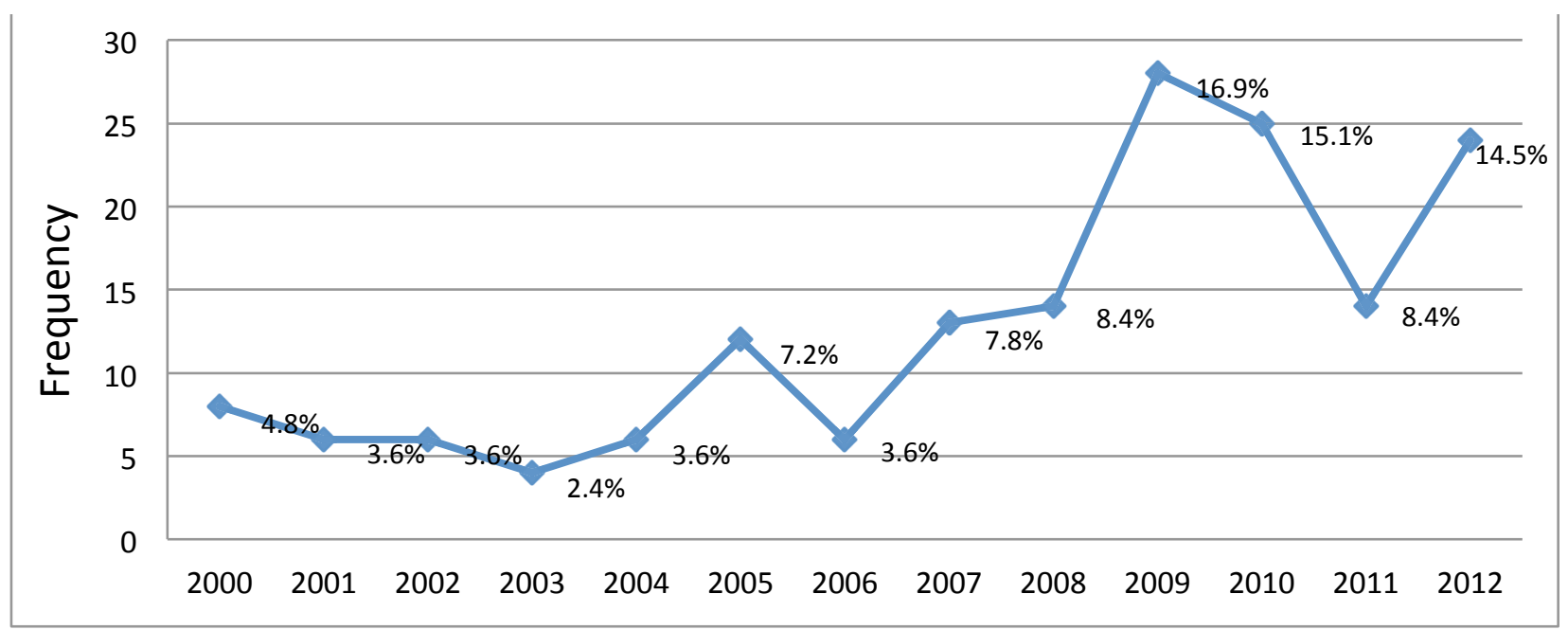

Figure 1. The number of research articles published on video learning every year

Based on these categories; the two researchers coded all the articles and discussed and solved any discrepancies. After the coding of the articles, a descriptive analysis of the results was performed in order to explore the growth and the trend of video learning research. In order to be able to observe any potential change on the characteristics of video learning research of the last years, we split our articles in two periods. The first period was from 2000 to 2006 and the second period from 2007 to 2012. After, we explored one by one all the categories among the two periods in order to identify the tendencies.

\section{VIDEO LEARNING RESEARCH AND MOOCS}

One can claim that the research on video learning has been growing in the last years. Based on our analysis we can verify this assumption, as we can see from figure 1 the number of articles published every year regarding videobased learning research has increased, especially after 2007. In order to make this growth more clear, we divided the period under investigation into two sub-periods (1st 2000-2006 and 2nd 2007-2012). Although the duration of the first period was one year more, the articles published in the second period were over 2 times that of the first (48 in the 1 st and 118 in the 2 nd period), indicating that the research on the field of video learning is becoming more and more important in the last years. However, we need to take into account that after 2005 mobile and social technologies has grown exponentially [9]. As such the rapid growth of video learning might be connected with that.

Regarding Massive Online Open Courses (MOOCs), the first course carrying the name MOOC was offered in 2008 , so this is new phenomenon. In general, there are two different types of MOOCs, which are known as cMOOCs and XMOOCs. cMOOCs are based on a philosophy of connectivism and networking [10]. cMOOCs are quite distinct from the xMOOCs which are being developed by institutions and follow a more behaviourist approach. In that article, we focus on the more recent XMOOCs (hereinafter MOOCs) that are most of the times video based.

MOOC is the educational buzzword of the last two years on education [11]. The incremental growth of MOOCs came when the US elite institutions adopted them. Currently many of the top institutions on all over the world offer their courses through MOOCs [3]. Recent- ly we also saw MOOCs in non-English languages like French, Spanish, Chinese and Italian. However, no connection has been made among the recently appeared MOOCs and the well-established research on video learning, even though MOOCs are mostly video based. This is exactly the point where our paper contributes with an attempt to investigate MOOCs through the lens of videobased learning prior research.

\section{A. Portability}

During the last years eLearning tools have transited from fixed computer-based instructional media-such as desktop computers or even laptop computers - to mobile computer-based instructional media - such as tablets or even smart phones [12], a relevant issue concerns whether video based learning followed this trend. As such, we distinguished the studies on two sub-categories: the mobile and the stable devices used. As we can see from table 1 , stable devices are mostly used on video learning research, however research with mobile devices is incrementally growth in the second period. This is probably based on the proliferation of new mobile devices (e.g., tablets, smart phones) in the last years.

Besides the few years of MOOCs' existence, we saw successful applications of mobile technology on MOOCs. For instance MobiMOOC [13], although was not designed to be mobile, by using social media (i.e. twitter, google groups, and wikispaces) became mobile friendly with remarkable results in mobile learning.

TABLE I.

THE TECHNOLOGY OF VIDEO LEARNING SYSTEMS (PORTABILITY)

\begin{tabular}{|c|c|c|c|}
\hline PERIOD & Mobile & Stable & Unspecified* \\
\hline $2000-2012$ & $24(14.46 \%)$ & $108(65.06 \%)$ & $34(20.48 \%)$ \\
\hline $\begin{array}{c}1^{\text {st }} \text { Period }(2000- \\
2006)\end{array}$ & $1(2.08 \%)$ & $33(68.75 \%)$ & $14(29.17 \%)$ \\
\hline $\begin{array}{c}2^{\text {nd }} \text { Period }(2007- \\
2012)\end{array}$ & $23(19.49 \%)$ & $75(63.56 \%)$ & $20(16.95 \%)$ \\
\hline
\end{tabular}

* some of the studies do not belong on one of these categories or do not define the device, context type used etc. (e.g., literature reviews, conceptual, attitudinal) 


\section{B. Synchronization}

Another crucial factor on video learning is synchronization, as such we distinguished the studies among synchronous and asynchronous learner-instructor communication. Based on the results of table 2 and considering the evolution of technology it is obvious that the video learning research shifted to more asynchronous systems. Of course this can be explained by the time and other restrictions of synchronous systems. In addition this is in alignment with the lack of synchronization in the emerged learning technologies, including MOOCs.

\section{Interactivity}

The majority of the video learning studies have been conducted on asynchronous systems (table 2), however asynchronous systems can be distinguished on interactive and non-interactive (we consider synchronous systems as interactive). As we can see from table 3; there is an obvious trend to non-interactive systems. Although, on most of the systems as well as on MOOCs there are many ways of interaction among the system and the learner these interactions are detached from video learning system and belong to the general learning context (e.g., assessment, tutor communication). As such, we can admit that the video learning trend is in alignment with the design and the use of MOOCs.

\section{Type of Use}

Another important aspect is the type of video learning use; this is why we distinguished the studies by taking into account if the video was the main learning medium or only supplementary. As we can see from table 4 there is an equal distribution among the number of studies which are using videos as a main and supplementary learning medium, this equality applies on both first and second period. In addition, in both cases we have a growth on the number of articles; with a slightly bigger growth on the supplementary use. This growth on learning via supplementary video use is in alignment with MOOCs use, as although most of the times video is the central aspect of a MOOC, MOOCs are open, participatory and accessible online course that offers learners a way to connect, collaborate, and engage in the learning process through many and diverse ways [10].

\section{E. Context of use}

Regarding the context of video learning, we distinguished the studies on formal and informal context. As we can see from table 5 most of the studies are conducted on formal context, and this difference increases in the second period. Video based MOOCs have also been formalized and are able to give degrees. However, it is important to stress that cMOOCs are more informal and can be characterized as an open and distributed knowledge pool which can be used for flexible and ubiquitous learning.

\section{CONCLUSIONS}

This paper reviews the status and trend of video learning research of the last twelve years based on the articles published in eight major educational technology journals. In summary, the following table provides the taxonomy we used to categorize the video learning research and the tendency on each one of the sub-categories.

In brief, based on our analysis we can admit that:
- The number of peer-reviewed articles dealing with video learning has been significantly increased during the last years.

- Research with mobile devices has significantly increased during the last years.

- The video learning research has moved to more asynchronous and non-interactive systems.

Considering the large amount of research on video learning, future studies and developments on MOOCs can be benefited as MOOCs are mainly video based platforms.

TABLE II.

THE TECHNOLOGY OF VIDEO LEARNING SYSTEMS (SYNCHRONIZATION)

\begin{tabular}{|c|c|c|c|}
\hline Period & Synchronous & Asynchronous & Unspecified \\
\hline $2000-2012$ & $24(14.46 \%)$ & $114(68.67 \%)$ & $28(16.87 \%)$ \\
\hline $1^{\text {st }}$ Period & $9(18.75 \%)$ & $24(50.00 \%)$ & $15(31.25 \%)$ \\
\hline $2^{\text {nd }}$ Period & $15(12.71 \%)$ & $90(76.27 \%)$ & $13(11.02 \%)$ \\
\hline
\end{tabular}

TABLE III.

THE FEEDBACK OF VIDEO LEARNING SYSTEMS

\begin{tabular}{|c|c|c|c|}
\hline Period & Interactive & Non-interactive & Unspecified \\
\hline $2000-2012$ & $32(19.28 \%)$ & $102(61.45 \%)$ & $32(19.28 \%)$ \\
\hline $1^{\text {st }}$ Period & $13(27.08 \%)$ & $23(47.92 \%)$ & $12(25.00 \%)$ \\
\hline $2^{\text {nd }}$ Period & $19(16.10 \%)$ & $79(66.95 \%)$ & $20(16.95 \%)$ \\
\hline
\end{tabular}

TABLE IV.

TYPE OF LEARNING USE

\begin{tabular}{|c|c|c|c|}
\hline Period & $\begin{array}{c}\text { Main learning } \\
\text { medium }\end{array}$ & $\begin{array}{c}\text { Supplementary } \\
\text { learning medium }\end{array}$ & Unspecified \\
\hline $2000-2012$ & $60(36.14 \%)$ & $64(38.55 \%)$ & $42(25.30 \%)$ \\
\hline $1^{\text {st }}$ Period & $14(29.17 \%)$ & $13(27.08 \%)$ & $21(43.75 \%)$ \\
\hline $2^{\text {nd }}$ Period & $46(38.98 \%)$ & $51(43.22 \%)$ & $21(17.80 \%)$ \\
\hline
\end{tabular}

TABLE V.

CONTEXT OF LEARNING USE

\begin{tabular}{|c|c|c|c|}
\hline Period & Formal context & Informal context & Unspecified \\
\hline $2000-2012$ & $91(54.82 \%)$ & $37(22.23 \%)$ & $38(22.89 \%)$ \\
\hline $1^{\text {st }}$ Period & $23(47.92 \%)$ & $11(22.92 \%)$ & $14(29.17 \%)$ \\
\hline $2^{\text {nd }}$ Period & $68(57.63 \%)$ & $26(22.03 \%)$ & $24(20.34 \%)$ \\
\hline
\end{tabular}

TABLE VI.

CATEGORIES, SUB-CATEGORIES AND VIDEO LEARNING RESEARCH TENDENCY

\begin{tabular}{|c|c|c|}
\hline Categories & Sub-Categories & Tendency* \\
\hline Portability & Mobile & +++ \\
\hline & Stable & - \\
\hline Synchronization & Synchronous & - \\
\hline & Asynchronous & +++++ \\
\hline Interactivity & Interactive & - \\
\hline & Non-interactive & ++++ \\
\hline Type of use & Main & ++ \\
\hline & Supplementary & +++ \\
\hline Context of use & Formal & ++ \\
\hline & Informal & 0 \\
\hline
\end{tabular}

* every $5 \%$ difference among the $1^{\text {st }}$ and the second period depicted with one symbol ( + or - depending the nature of the difference); in the case of no difference we used 0 
In addition, although MOOCs are a new trend and are still under development, we can admit their association with portable and more flexible technologies [13]. Moreover, although synchronous and interactive systems have been refined in the last years, we can see a tendency to more asynchronous and non-interactive video systems. This is exactly the rationale of MOOCs, open, participatory, accessible and distributed knowledge pool which can be used for flexible learning [10].

These findings could be good references for those who plan to contribute to the video learning studies and the emerging area of MOOCs. In addition, the results of our analysis could be helpful to policy-decision makers in order to support the development of their educational direction.

The study provides some early insights of video learning research agenda and how this agenda might be useful for video based MOOCs, however in out attempt there are certain limitations. First, MOOCS started at 2008 (as nonvideo based platform) then in 2011-12

As such there are only few academic studies on videobased MOOCs as there has been no time for systematic research on them. Second, the rise of videos learning research is noticed at 2007 (Figure 1); as such this rise might be based on new video platforms launch (e.g., YouTube started in 2005) or even at the exponential growth of mobile and social technologies at 2005 [9]. So, although video learning research has much to offer on video-based MOOCs; their links are very weak because of the little lifetime of MOOCs. Another limitation of the current study is the massive and epidermic analysis of the papers.

Besides the aforementioned limitations, this first investigation provides valuable insights and opens new avenues for future work. In the next step of this ongoing work we will proceed on further analysis of the collected articles by discarding the opinion papers, literature reviews, short papers, and non-empirical papers. In addition, we will consider separating papers dealing with video learning via real time video communication with the instructor; as we want to focus our study more on the learning aspects of video and not on the communication ones.

\section{ACKNOWLEDGMENT}

The authors wish to thank the helpful comments and suggestions from reviewers.

\section{REFERENCES}

[1] R. H. Kay. "Exploring the use of video podcasts in education: a comprehensive review of the literature". Computers in Human Behavior, 2012, 28(3), 820-831. http://dx.doi.org/10.1016/j.chb. 2012.01.011

[2] M.N. Giannakos, K. Chorianopoulos, M. Ronchetti, P. Szegedi, and S.D. Teasley, "Analytics on video-based learning" In Proceed- ings of the Third International Conference on Learning Analytics and Knowledge (LAK '13), ACM, 2013, pp. 283-284.

[3] F.G. Martin. "Will massive open online courses change how we teach?" Commun. ACM 55, 8, 2012, pp. 26-28.

[4] Corbyn, Z. (2012). This could be huge... Times Higher Education. http://www.timeshighereducation.co.uk/story.asp? sectioncode $=26$ \&storycode $=422034 \& \mathrm{c}=1$ accessed 2013-09-21

[5] C. Brooks. et al., "OpenCast Matterhorn 1.1: reaching new heights". In Proceedings of MM '11, ACM, 2011, pp. 703-706.

[6] M.N. Giannakos. "Exploring the video-based learning research: A review of the literature". British Journal of Educational Technology, 44(6), 2013, pp. 191-195. http://dx.doi.org/10.1111/bjet.12070

[7] G.-J. Hwang, \& P.-H. Wu, "Advancements and trends in digital game-based learning research: a review of publications in selected journals from 2001 to 2010" British Journal of Educational Technology, 43(1), 2012, pp. 6-10. http://dx.doi.org/10.1111/j.14678535.2011.01242.x

[8] Y.-C. Hsu. H.N.J. Ho, C.-J. Tsai, G.-J. Hwang, H.-C. Chu, C.-Y. Wang, \& N.-S. Chen. "Research trends in technology-based learning from 2000 to 2009: A content Analysis of publications in selected journals" Educational Technology \& Society, 15 (2), 2012, pp. 354-370.

[9] L. Johnson, A. Levine, R. Smith, \& S. Stone, The 2010 Horizon Report, The New Media Consortium, Austin, 2010. TX.

[10] G. Siemens. "MOOCs are really a platform" eLearnspace. 2012, http://www.elearnspace.org/blog/2012/07/25/moocs-are-really-aplatform/ accessed 2013-02-21

[11] EDUCAUSE Learning Initiative. (2011). "Seven things you should know about MOOCs" Retrieved from http://net.educause.edu/ir/library/pdf/ELI7078.pdf

[12] E. Sung, \& R.E. Mayer, "Students' beliefs about mobile devices versus personal computers in South Korea and the United States" Computers and Education, 59(4), 2012, pp. 1328-1338. http://dx.doi.org/10.1016/j.compedu.2012.05.005

[13] I. de Waard, S, Abajian, M. S. Gallagher, R. Hogue, N. Keskin, A. Koutropoulos, et al. "Using mLearning and MOOCs to understand chaos, emergence, and complexity in education," International Review of Research in Open and Distance Learning, 2011, 12(7), pp. 94-115.

\section{AUTHORS}

Michail N. Giannakos is ERCIM/Marie Curie Fellow in the Department of Computer and Information Science at Norwegian University of Science and Technology (NTNU), NO-7491, Trondheim, Norway (e-mail: michailg@idi.ntnu.no).

Letizia Jaccheri is a Professor of Software Engineering in the Department of Computer and Information Science at Norwegian University of Science and Technology (NTNU), NO-7491, Trondheim, Norway (e-mail: letizia@idi.ntnu.no).

John Krogstie is a Professor of Information Systems in the Department of Computer and Information Science at Norwegian University of Science and Technology (NTNU), NO-7491, Trondheim, Norway (e-mail: krogstie@idi.ntnu.no)

Submitted, June, 19, 2013. Published as resubmitted by the authors on October, 31, 2013. 